\title{
ESTUDIO ETNOBOTÁNICO DE PLANTAS MEDICINALES E IMPORTANCIA DE CONSERVAR LAS ESPECIES VEGETALES SILVESTRES DEL CANTÓN CHILLA, ECUADOR
}

\author{
ESTUDO ETNOBOTÂNICO DE PLANTAS MEDICINAIS E A IMPORTÂNCIA DA \\ CONSERVAÇÃO DAS ESPÉCIES DE PLANTAS SILVESTRES \\ DO CANTÃO DO CHILLA, EQUADOR
}

\author{
Jonathan Bladimir ZHIMINAICELA-CABRERA*; José Nicasio QUEVEDO-GUERRERO; Sayda Noemi HERRERA-REYES; Angel \\ Roberto SÁNCHEZ-QUINCHE; Laura Yadira BERMEO-GUALAN
}

Facultad de Ciencias Agropecuarias, Granja Experimental Santa Inés, Universidad Técnica de Machala, Ecuador. *jzhiminai1@utmachala.edu.ec; jquevedo@utmachala.edu.ec; sherrera@utmachala.edu.ec; arsanchez@utmachala.edu.ec; lbermeo3@utmachala.edu.ec

\section{RESUMEN}

El objetivo de este estudio fue identificar las especies vegetales silvestres y cultivadas que utilizan las comunidades rurales del cantón Chilla, provincia de El Oro-Ecuador, con la finalidad de conocer los conocimientos ancestrales, en cuanto al uso de plantas medicinales e indagar la resiliencia que presentan estas especies vegetales a condiciones atmosféricas adversas, que en general en esta área son las heladas y sequías producidas por el cambio climático. Para ello, se realizó un estudio de tipo cuantitativo etnográfico, observacional y trasversal. Desarrollado en 16 comunidades rurales del Cantón Chilla-Ecuador, con encuestas semiestructuradas e indagatorias evaluando a 83 pobladores con un rango de edad 20-70 años, 44\% mujeres y $56 \%$ hombres. Se identificaron 92 especies vegetales, 59 exóticas y 33 nativas, presentes en 45 familias y 83 géneros. La categoría con más importancia fue: uso medicinal - respiratorio $26.81 \%$ y gastrointestinal $25.49 \%$. El método de preparación fue: infusión $84 \%$. Parte de la planta utilizada: $55 \%$ hojas. Mecanismo de administración: $78 \%$ bebida. El habitad: $46 \%$ huertos. Las especies silvestres presentan mejor resiliencia a condiciones atmosféricas adversas infiriendo se deba a la huella genética, pero tienen menor uso en la población evaluada, debido a que plantas se encuentran en sitios inhóspitos, dificultando su adquisición. Se debe considerar medidas para proteger y conservar estas especies y sus características, ante la problemática de la expansión agrícola que propiciara la erosión genética de estas especies vegetales con características únicas para el posible desarrollo de fitofármacos. Además, se determinó que el $98 \%$ de los encuestados se identificaron como mestizo considerándose un aporte cultural pues se adjudica que parte de la protección vegetal es en su mayoría la etnia indígena.

Palabras-clave: conocimientos ancestrales; conservación de los recursos; etnobotánica; cambio climático; etnobiología.

\section{RESUMO}

O objetivo deste estudo foi identificar as espécies de plantas silvestres e cultivadas utilizadas pelas comunidades rurais no cantão de Chilla, província de El Oro-Equador, a fim de conhecer os conhecimentos ancestrais sobre o uso de plantas medicinais e investigar a resiliência destas espécies vegetais às condições atmosféricas adversas, que em geral nesta área são geadas e secas causadas pelas mudanças climáticas. Para tanto, foi realizado um estudo etnográfico quantitativo, observacional e de corte transversal. Foi realizada em 16 comunidades rurais do cantão Chilla-Equador, com pesquisas semiestruturadas e investigativas avaliando 83 habitantes com uma faixa etária de 20-70 anos, 44\% mulheres e 56\% homens. Identificadas 92 espécies de plantas, 59 exóticos e 33 nativos, presentes em 45 famílias e 83 gêneros. A categoria mais importante foi: uso medicinal - respiratório $26,81 \%$ e gastrointestinais $25,49 \%$. O método de preparação foi: infusão $84 \%$. Parte da planta utilizou: $55 \%$ de folhas. Mecanismo de administração: $78 \%$ bebida. O habitat: $46 \%$ dos pomares. As espécies silvestres apresentam melhor resiliência às condições atmosféricas adversas que infectam é devido à impressão digital genética, mas têm menos uso na população avaliada, pois as plantas estão em locais inóspitos, o que dificulta a aquisição. Devem ser consideradas medidas para proteger e conservar estas espécies e suas características, dado o problema da expansão agrícola que promoveria a erosão genética destas espécies vegetais com características únicas para o possível desenvolvimento de fitofármacos. Além disso, foi determinado que $98 \%$ dos pesquisados se identificaram como mestiços, considerando-se uma contribuição cultural, uma vez que se afirmar que parte da proteção das plantas é principalmente o grupo étnico indígena.

Palavras-chave: conhecimento ancestral; conservação de recursos; etnobotânica; mudança climática; etnobiologia. 


\section{INTRODUCCIÓN}

La etnobotánica es la ciencia que estudia el uso de la flora y la interacción con seres humanos. Se han identificado que diversas especies vegetales, por sus compuestos bioactivas, son utilizadas para contrarrestar dolencias o enfermedades para la salud humana, y también para el desarrollo de fitofármacos (BERMÚDEZ; OLIVEIRA-MIRANDA; VELÁZQUEZ, 2005). Los usos botánicos de las plantas en Ecuador se utilizan de diversas formas tanto espiritual, medicinal y cultural (BUSSMANN; SHARON, 2014). De las cuales se conocen diversos usos fitoterapéuticas (DE LA TORRE; MACÍA, 2008). En general su uso se debe a los conocimientos ancestrales, los cuales se ven amenazados en algunas etnias ecuatorianas por factores como la colonización, la falta de documentación y los procesos de modernización (ARMIJOS; COTA; GONZÁLEZ, 2014).

De acuerdo a la Organización de las Naciones Unidas y el protocolo de NAGOYA para la protección de especies y la Agenda 2030 es importante conservar las especies en su habitad natural y desarrollar un sistema de salud equitativos, por medio de métodos convencionales o alternativos. De ahí la importancia del estudio de las plantas medicinales y su conservación pues la expansión de las fronteras agrícolas han ocasionado la perdida de especies vegetales (BUSSMANN, 2002), que pudieran tener huellas genéticas en su genoma que le permitieran la resiliencia a condiciones atmosféricas y agronómicas adversas (ACOSTA DE LA LUZ, 2013a).

Su utilización nace de la necesidad e indisponibilidad de medicamentos sintéticos en comunidades rurales en desarrollo, donde el acceso es restringido a los medicamentos en droguerías debido a condiciones topográficos o demográficos (BUSSMANN; SHARON, 2014). En Ecuador debido a la mega diversidad de especies, producto de las condiciones climáticas y los diversos pisos latitudinales en una misma región, permiten la adaptación de especies introducidas extranjeras y el desarrollo de características únicas de especies vegetales naturales en condiciones atmosféricas no idóneas (DE LA TORRE; MACÍA, 2008).

Las plantas medicinales por las comunidades rurales de muchas regiones de Ecuador son utilizadas como fitoterapéuticas, pero, aunque se hayan realizado estudios de las propiedades bioactivas de algunas especies para el cuidado de la salud, existen especies de las cuales no se tiene conocimiento, muchas de ellas de origen silvestre que son las más utilizadas, pero menos comercializadas (BUSSMANN; SHARON, 2014). Además, no se conoce la variabilidad de sus principios activos al habitar en condiciones atmosféricas y agronómicas adversas, producidas por la variabilidad que produce el cambio climático, las cuales influirán en la fisiología y composición química de la especie vegetal (YEPES, ADRIANA; SILVEIRA BUCKERIDGE, 2011). Especialmente en comunidades donde las condiciones atmosféricas son variantes, permitiendo mayor cantidad de cambios en la fisiología natural de las plantas (ACOSTA DE LA LUZ, 2013b).

En la zona sur del Ecuador, especialmente en Loja, las comunidades indígenas históricamente han protegido y permitido la conservación de la biodiversidad y la seguridad alimentaria (BATISTA; MILIOLI; CITADINI-ZANETTE, 2020), caso contrario a la provincia del El Oro donde solo se ha realizado un estudio de este tipo no documental de las especies (CHAMBA et al., 2019). En Chilla, debido a la actividad agropecuaria, se han degradado los suelos y el ambiente con sus especies (BELDUMA BELDUMA et al., 2020). Por esta razón, el objetivo de este estudio fue identificar el uso medicinal de las plantas del Cantón Chilla, El Oro-Ecuador e indagar en los conocimientos ancestrales de acuerdo a su origen. Con la finalidad de vislumbrar las respuestas acerca de las especies vegetales de acuerdo a su origen ante el cambio climático, con un enfoque para la conservación de la diversidad genética y la protección de los ecosistemas naturales.

\section{MATERIALES Y MÉTODOS}

La presente investigación se realizó en 16 comunidades rurales del Cantón Chilla, Provincia de El Oro, Ecuador; a $03^{\circ} 27^{\prime} 30^{\prime \prime}$ latitud sur y $79^{\circ} 35^{\prime} 52^{\prime \prime}$ longitud Noreste, con una altitud de 200-3.610 msnm (Figura 1).

La extensión es de $289 \mathrm{~km}^{2}$, sus límites son al norte Cantón Pasaje, al sur con los cantones de Zaruma y Atahualpa, al este cantón Zaruma. El cantón Chilla contiene diversos suelos de acuerdo a su altitud, en general son suelos francos arcillo arenosos, limosos, arcillosos arenosos, arenosos, con unos tres tipos de climas que oscilan entre $8^{\circ} \mathrm{C}-12^{\circ} \mathrm{C}$ máxima altitud, $12^{\circ} \mathrm{C}-15^{\circ}$ altitud media y altitud baja de $20^{\circ} \mathrm{C}-30^{\circ} \mathrm{C}$. 

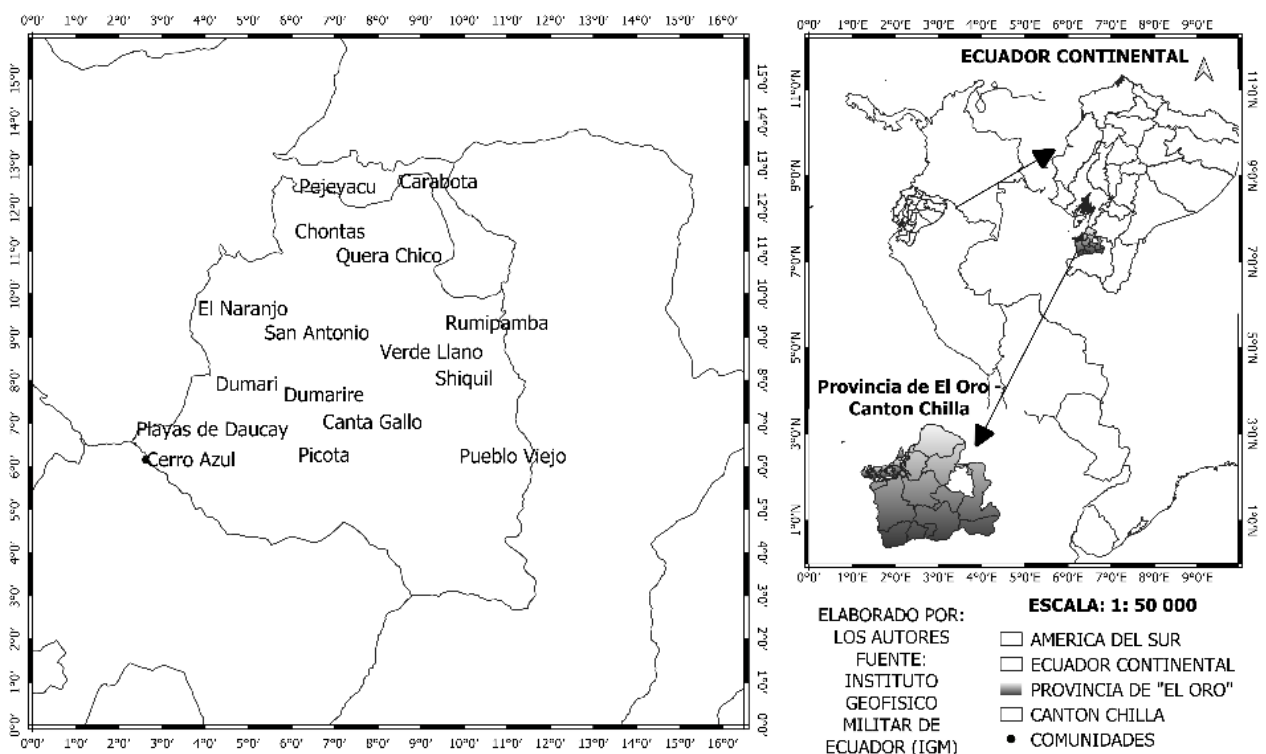

Figura 1. Localización de las comunidades rurales donde se realizaron las encuestas en el Cantón Chilla, Provincia de El Oro, Ecuador.

\section{Trabajo de Campo}

El estudio etnobotánica se llevó a cabo entre enero y abril del 2020, siguiendo un método cualitativo etnográfico, con breves cambio en la metodología de Zambrano-Intriago et al. (2015). La zona de estudio fue identifica de acuerdo a la población que habita en esas comunidades intercalando comunidades con poblaciones extensas y con poblaciones pequeñas de área rural que representa un $58.7 \%$ de los 2500 habitantes: se tomaron 16 puntos del cantón chilla, para ello se les informo a los pobladores la finalidad del estudio y la vital importancia de indicar con responsabilidad la información, las poblaciones en marzo y abril fueron tomadas por medios digitales debido a las restricciones de circulación. Para determinar el tamaño de la muestra se elaboró con la formula general y de acuerdo a las indicaciones de García-García et al. (2013).

Los puntos fueron identificados por medio de mapas cartográficos del Instituto Geográfico Militar del Ecuador, atreves del Geoportal a una escala 1:50000, donde se evaluaron a 83 viviendas con un promedio de 1-2 encuestado con un promedio de edad 20-70 años, que expresaron tener conocimientos del uso de plantas medicinales. Se tomó el punto de referencia de ahí en adelante un mínimo de 3-5 de las casas más próximas. Se emplearon encuestas semiestructuradas donde se indaga cuáles son las plantas utilizadas para tratar dolencias o enfermedades, uso común, método de preparación, vía de administración y órgano de la planta.

\section{Clasificación de las enfermedades}

La clasificación de las enfermedades tratadas tradicionalmente con plantas medicinales fue determinada de acuerdo a Zambrano-Intriago et al. (2015), modificado de acuerdo a las indicaciones de los encuestados y tomando en cuenta las consideraciones que presenta la Clasificación de Internacional de Enfermedades (CIE 10).

\section{RESULTADOS Y DISCUSIÓN}

Las especies más representativas fueron en Laminaceae (14 especies y 12 géneros) y Asteraceae (12 especies y 12 géneros) semejantes a la provincia de Imbabura (FERNÁNDEZ-CUSIMAMANI et al., 2019). Para las demás plantas medicinales el promedio de especies y géneros fue de $2 \pm 1$. La familia Laminaceae se encuentra con frecuencia en los estudios etnobotánicos de Ecuador (Figura 2).

\section{Características sociales de la población encuestada}

Se realizaron 83 encuestas de las cuales fueron: femenino $53.01 \%$ y masculino $46.99 \%$, la etnia con la que se identifican mestizos $98.80 \%$ y blanco $1.20 \%$. La ocupación: Agricultor $38.55 \%$, Cuidado del hogar $25.30 \%$, Estudiante $18.07 \%$, Servidor público $12.05 \%$, Obrero $6.02 \%$. El grado de educación académico: Secundaria 43.37\%, Primeria 26.51\%, Pregrado 20.48\%, Ninguna 9.64\%; no se 
encontró encuestados con estudios de postgrado. Edad $45.78 \%$ de 20 a 30 años, $20.48 \%$ de $31-40$, $14.46 \%$ de $41-50,8.43 \%$ de $51-60,10.84 \%$ de $61-70$.

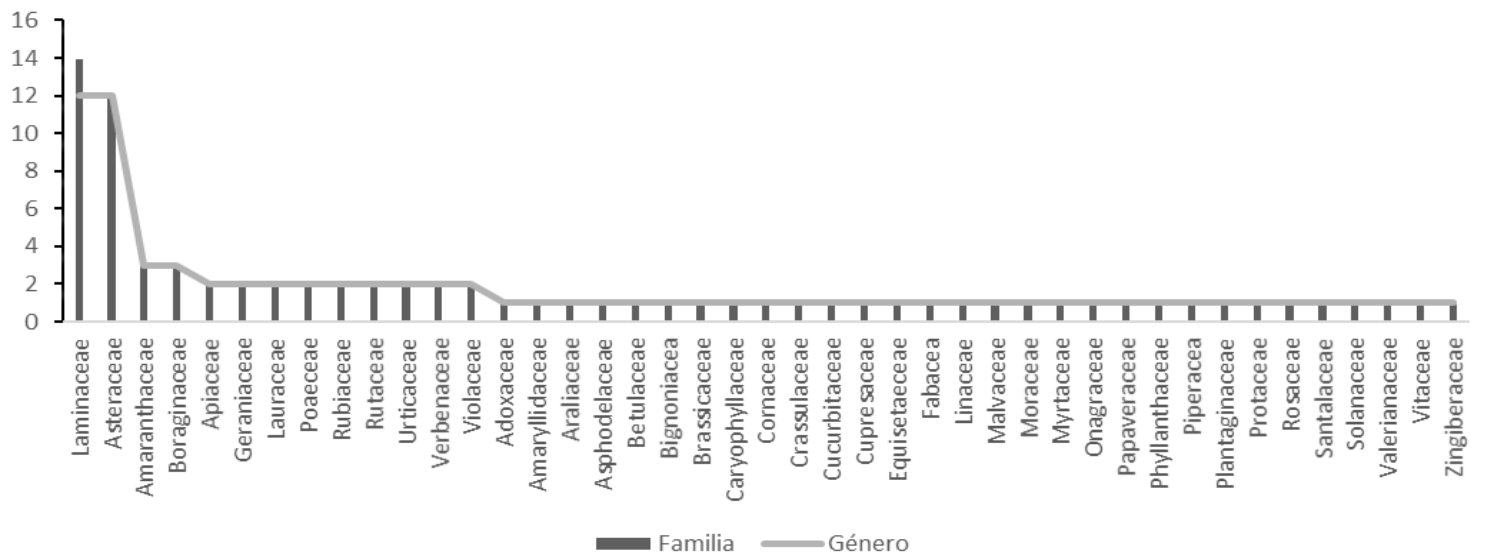

Figura 2. Diversidad de familias de plantas medicinales utilizadas por los habitantes del cantón Chilla, Provincia de El Oro, Ecuador.

\section{Plantas medicinales utilizadas}

De acuerdo al estudio se determinó dos orígenes de las cuales recurren los encuestados para la utilización de plantas medicinales $65 \%$ exóticas y $35 \%$ nativas valores cercanos a los reportados (BUSSMANN; SHARON, 2014), las especies vegetal nativas tuvieron similitud a los Cusco (HUAMANTUPA et al., 2011) con quienes se comparten similitudes en flora y en comunidades regiones andinas de Ecuador (ANSALONI et al., 2010). En el 78\% de los casos la medicina tradicional se utiliza para tratar eventos leves en las enfermedades y $22 \%$ tratamiento continuo, encontrándose similitud a la comunidad rural de Babahoyo (GALLEGOS ZURITA, 2016). La utilización de las plantas medicinales en Chilla, tienen menor uso y por ende menor interés para su conservación promoviendo la erosión genética (ARIAS TOLEDO; TRILLO; GRILLI, 2010). Se describen de acuerdo a la frecuencia de uso las características de la utilización de las plantas medicinales (ANEXO 1).

\section{Categoría de Uso}

En la Tabla 1 se presentan las enfermedades características, que de acuerdo a los encuestados son tratadas, se toma como referencia el CIE 10, para categorizar; los resultados que se presentan son: Respiratorio 26.81\%, Gastrointestinal 25.49\%, Nervioso $10.77 \%$, Urológico $8.79 \%$, Otros/Todo 7.91\%, Piel 5.71\%, Renal 3.96\%, Sanguíneo 3.52\%, Inmunológico 3.08\%, Osteomuscular 1.76\%, Neurológico $0.88 \%$, Hepático $0.88 \%$, Sistema sensorial $0.44 \%$. Resultados con variaciones en el uso respiratorio de Gallegos Zurita (2016) y semejantes a Ansaloni et al. (2010).

Tabla 1. Enfermedades tratadas con plantas medicinales por los habitantes del cantón Chilla, El Oro-Ecuador.

\begin{tabular}{llc}
\hline $\begin{array}{l}\text { Sistema del cuerpo } \\
\text { (categorías) }\end{array}$ & Enfermedades o afecciones & \% de uso \\
\hline Respiratorio & $\begin{array}{l}\text { Dificultad para respirar, dolor de garganta, dolor de pecho, bronquitis, tos y } \\
\text { resfríos. }\end{array}$ & $26.81 \%$ \\
Gastrointestinal & Diarrea, vómitos, dolor de estómago, gastritis, parásitos y cólicos estomacales. & $25.49 \%$ \\
& & $10.77 \%$ \\
Nervioso & Enfermedad de Parkinson, estrés y nervios. & $8.79 \%$ \\
Urológico & Infección del tracto urinario, diurético y desinflamación de la próstata. & $7.91 \%$ \\
Otros/Todo el cuerpo & Fiebre, dolor de cabeza, vértigo / mareos, dejar de sudar, diabetes, mordedura \\
& de serpiente, “dolor de hinchazón", caída del cabello, desinflamación y para & $5.71 \%$ \\
Piel & bajar de peso. & $3.96 \%$ \\
Renal & Infecciones, heridas e inflamaciones en la piel y granos. & $3.52 \%$ \\
Sanguíneo & Enfermedades renales. & $3.08 \%$ \\
Inmunológico & Colesterol, presión arterial alta y la circulación sanguínea. &
\end{tabular}




\begin{tabular}{|c|c|c|}
\hline $\begin{array}{l}\text { Sistema del cuerpo } \\
\text { (categorías) }\end{array}$ & Enfermedades o afecciones & $\%$ de uso \\
\hline Osteomuscular & $\begin{array}{l}\text { Fracturas óseas, dolor de huesos, enfermedades óseas, dolor en las } \\
\text { articulaciones-cintura, inflamación del cuerpo, reumatismo, dolor de cuello y } \\
\text { extremidades. }\end{array}$ & $1.76 \%$ \\
\hline Neurológico & Adormecimiento de las extremidades y parálisis. & $0.88 \%$ \\
\hline Hepático & Enfermedades hepáticas e hígado graso. & $0.88 \%$ \\
\hline Sistema sensorial & Dolores en nariz, ojos, oídos, sinusitis, y dolor dental. & $0.44 \%$ \\
\hline
\end{tabular}

\section{Vía de administración y método de preparación}

Las vías de administración para dar un enfoque más general de sus usos se pueden clasificar en vía oral: entre ellas bebida, Inhalación y Comida o la vía tópica: uso externo y baño (Figura 3). Los cuales tiene relación lógica debido a que es el mecanismo por el cual se trata la enfermedad en todos los casos la utilización de agua para las preparaciones sobre sale (Figura 4), en todos los sentidos debido a que las altas temperaturas permiten obtener los compuestos, aunque su utilización es empírica es uno del mecanismo más utilizados para extraer compuestos de plantas medicinales (BUSSMANN, 2002). Los resultados presentaron similitud a la comunidad rural del cantón Quevedo en estas dos variables evaluadas (ZAMBRANO-INTRIAGO et al., 2015). Se alude al uso de las plantas medicinales aromáticas con utilización medicinal, que utilizan como acompañamiento alimenticio en estas comunidades rurales. Las plantas con mayor resiliencia de acuerdo a los pobladores encuestados son las plantas silvestres o nativas, estos resultados se pueden deber a la capacidad de adaptarse a condiciones adversas especialmente cuando son especies locales (ALTIERI; NICHOLLS, 2008), en el caso de las plantas medicinales también se cumple (RIVAS, 2014), corroborando lo indicado por los encuestados.

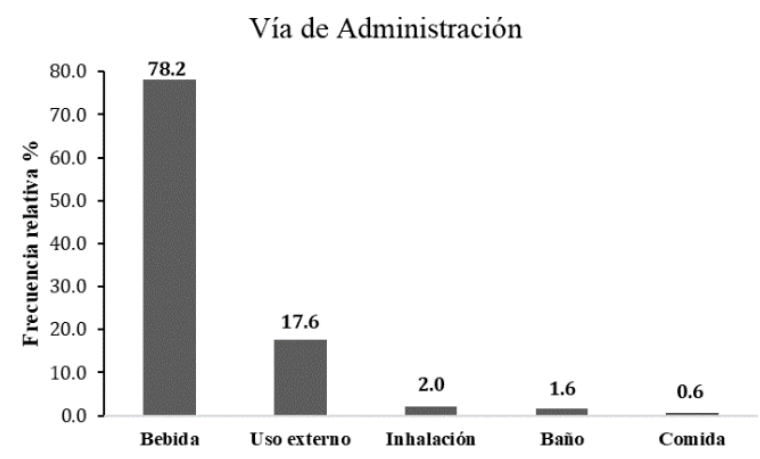

Figura 3. Vía de Administración.

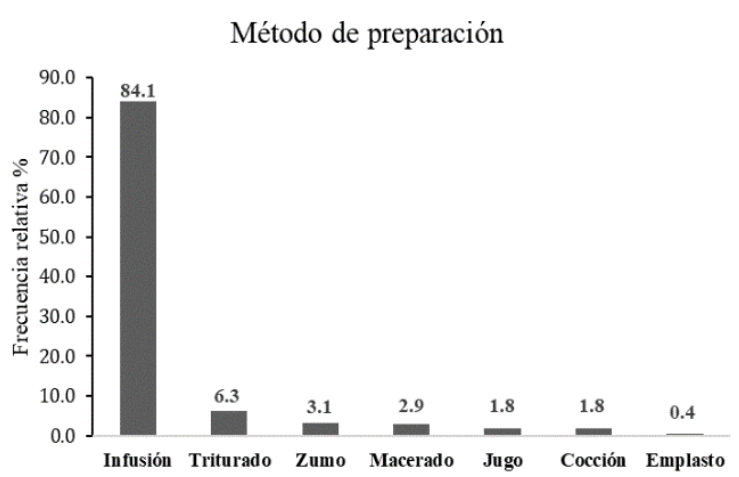

Figura 4. Método de preparación.

\section{Parte de la planta}

Las hojas en general, son los órganos más utilizados en la Zona Sur del Ecuador para uso medicinal (Figura 5), no se encontró variaciones con los estudios realizados en Ecuador (ANSALONI et al., 2010).

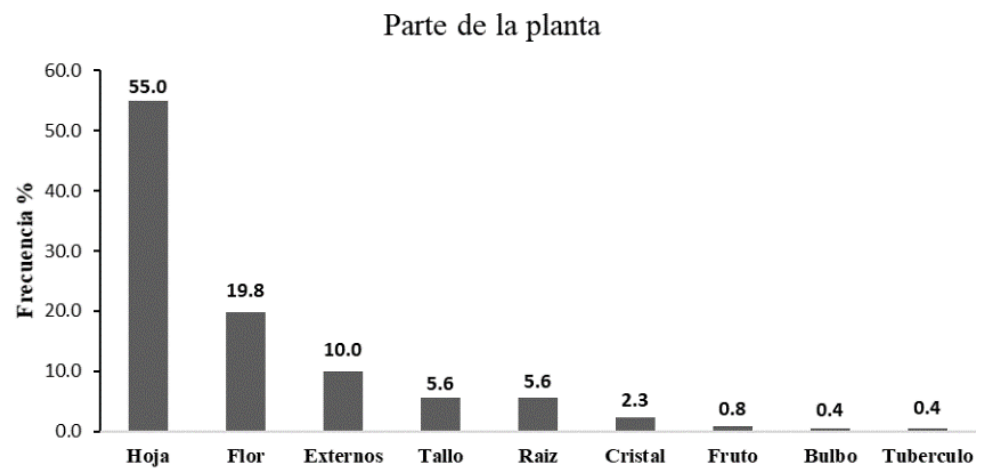

Figura 5. Parte u órgano de la planta. 


\section{CONCLUSIONES}

Se evidencio la influencia que ejerce la medicina tradicional en la zona sur del Ecuador, no solo en las etnias indígenas, sino también en la mestiza, etnia aborigen que surge de la mezcla entre pueblo indígenas y conquistadores españoles, pues el 98\% de la población encuestada en Chilla, se identificaron como mestizos. Los usos en las plantas medicinales del cantón Chilla, tuvieron relación a estudios desarrollados en Ecuador y Perú en la región de altos andinos, especialmente por la relación cultural y condiciones geográficas y climáticas que compartimos al estar ubicado en la Zona Sur de Ecuador el estudio.

Las comunidades rurales en la parte alta intercambian el material vegetal en menor cantidad a la comercialización, a excepción de las especies que no se adaptan en la zona, la preferencia es asociar las plantas medicinales con sus cultivos.

Las plantas medicinales resilientes al cambio climático son las silvestres y en menor medida cuando se asocia a otros cultivos agrícolas. Las pérdidas se dan por las heladas y en menor medida sequias, se infiere debido a la huella genética que permite adaptarse a las condiciones climáticas cambiantes, estos resultados se presentan similitud especialmente con las especies nativas del Norte de Perú. Actualmente, no existe herbarios que protejan la diversidad genética de las especies medicinales de esta zona, o recursos de importancia para la seguridad alimentaria.

\section{AGRADECIMIENTOS}

Los autores agradecen al municipio y a las comunidades del Cantón Chilla, la Universidad Técnica de Machala, por permitir los espacios para el desarrollo de investigaciones regionales. Especialmente a Sr. Porfirio Orellana, quien ayudo con su jardín botánico de plantas medicinales, dio a conocer el ambiente de crecimiento y sus experiencias con plantas de origen silvestres. Ha Sueny Paloma Lima do Santos por la traducción del manuscrito. Además, a los revisores pares editores de esta revista por sus comentarios, que ayudaron a mejorar este trabajo.

\section{REFERENCIAS}

ACOSTA DE LA LUZ, L. Principios agroclimáticos básicos para la producción de plantas medicinales.

Revista Cubana de Plantas Medicinales, v. 8, n. 1, 2013a.

ACOSTA DE LA LUZ, L. Principios agroclimáticos básicos para la producción de plantas medicinales.

Revista Cubana de Plantas Medicinales, v. 8, n. 1, 2013b.

ALTIERI, M. A.; NICHOLLS, C. Los impactos del cambio climático sobre las comunidades campesinas y de agricultores tradicionales y sus respuestas adaptativas. Agroecología, v. 3, p. 7-24, 2008.

ANSALONI, R. et al. Estudio Preliminar sobre Plantas Medicinales Utilizadas en Algunas Comunidades de las Provincias de Azuay, Cañar y Loja, para Afecciones del Aparato Gastrointestinal. Revista Tecnológica ESPOL, v. 23, n. 1, p. 89-97, 2010.

ARIAS TOLEDO, B.; TRILLO, C.; GRILLI, M. Uso de plantas medicinales en relación al estado de conservación del bosque en Córdoba, Argentina. Ecología austral, v. 20, n. 3, p. 235-246, 2010.

ARMIJOS, C.; COTA, I.; GONZÁLEZ, S. Traditional medicine applied by the Saraguro yachakkuna: a preliminary approach to the use of sacred and psychoactive plant species in the southern region of Ecuador. Journal of Ethnobiology and Ethnomedicine, v. 10, n. 1, p. 26, 2014.

BATISTA, K. M.; MILIOLI, G.; CITADINI-ZANETTE, V. Referência de uso e conservação da biodiversidade: considerações teóricas sobre o povo Mbya Guarani. Ethnoscientia, v. 5, n. 1, p. 37-44, fev. 2020.

BELDUMA BELDUMA, R. G. et al. Gestión y uso del suelo agropecuario en la zona de rural del Cantón Chilla desde una visión socioeconómica. Revista Universidad y Sociedad, v. 12, n. 1, p. 299-306, 2020.

BERMÚDEZ, A.; OLIVEIRA-MIRANDA, M. A.; VELÁZQUEZ, D. La investigación etnobotánica sobre plantas medicinales: una revisión de sus objetivos y Actuales Enfoques. Interciencia, v. 30, n. 8, p. 453-459, 2005a.

BERMÚDEZ, A.; OLIVEIRA-MIRANDA, M. A.; VELÁZQUEZ, D. La Investigación Etnobotánica Sobre Plantas Medicinales: Una Revisión De Sus Objetivos Y Enfoques Actuales Alexis Bermúdez, María a . Oliveira-Miranda. Interciencia, v. 30, n. 8, p. 453-459, 2005 b.

BUSSMANN, R.; SHARON, D. Two decades of ethnobotanical research in Southern Ecuador and Northern Peru. Ethnobiology and Conservation, v. 2014, p. 1-50, jan. 2014.

BUSSMANN, R. W. Ethnobotany and Biodiversity Conservation. In: Modern Trends in Applied Terrestrial Ecology. Boston, MA: Springer US, 2002. p. 343-360.

CHAMBA, M. J. T. et al. Conocimientos, Actitude y prácticas de la medicina ancestrak en la población de

Casacay Ecuador. Revista Tzhoecoen, v. 11, n. 4, p. 88-94, 2019.

DE LA TORRE, L.; MACÍA, M. J. La etnobotánica en el Ecuador. In: Enciclopedia de las Plantas Útiles 
del Ecuador. [s.l: s.n.]. p. 1-15.

FERNÁNDEZ-CUSIMAMANI, E. et al. Estudio etnobotánico de plantas medicinales utilizadas en tre cantones de la provincia Imbabura, Ecuador. Agrociencia, v. 53, n. 5, p. 797-810, 2019.

GALLEGOS ZURITA, M. Las plantas medicinales: principal alternativa para el cuidado de la salud, en la población rural de Babahoyo, Ecuador. Anales de la Facultad de Medicina, v. 77, n. 4, p. 327, 2016.

GARCÍA-GARCÍA, J. A.; REDING-BERNAL, A.; LÓPEZ-ALVARENGA, J. C. Cálculo del tamaño de la muestra en investigación en educación médica. Investigación en Educación Médica, v. 2, n. 8, p. 217-224, out. 2013.

HUAMANTUPA, I. et al. Riqueza, uso y origen de plantas medicinales expendidas en los mercados de la ciudad del Cusco. Revista peruana de biología, v. 18, n. 3, p. 283-292, 2011.

RIVAS, G. Huertos familiares: para la conservación de la agrobiodiversidad, la promoción de la seguridad alimentaria y la adaptación al cambio climático. Ambientico, n. 243, p. 4-10, 2014.

YEPES, ADRIANA; SILVEIRA BUCKERIDGE, M. Plant responses to meteorological events related to climate change - review. Colombia Forestal, v. 14, n. 0120-0739, p. 213-232, 2011.

ZAMBRANO-INTRIAGO, L. F. et al. Estudio etnobotánico de plantas medicinales utilizadas por los habitantes del área rural de la Parroquia San Carlos, Quevedo, Ecuador. Universidad y Salud, v. 17, n. 1, p. $97-$ $111,2015$. 


\section{Anexo 1}

Tabla 2. Plantas medicinales utilizadas tradicionalmente por las comunidades del cantón Chilla, El OroEcuador.

\begin{tabular}{|c|c|c|c|c|c|c|c|}
\hline $\begin{array}{l}\text { Nombre } \\
\text { común }\end{array}$ & $\begin{array}{l}\text { Nombre } \\
\text { científico }\end{array}$ & Familia & Uso medicinal & $\begin{array}{l}\text { Método } \\
\text { preparación }\end{array}$ & $\begin{array}{c}\text { Parte } \\
\text { utilizada }\end{array}$ & $\begin{array}{c}\text { Vía } \\
\text { administración }\end{array}$ & Origen \\
\hline Manzanilla & $\begin{array}{l}\text { Matricaria chamomilla } \\
\text { L. }\end{array}$ & Asteraceae & $\begin{array}{l}\text { Dolor estomacal, } \\
\text { antinflamatorio, Acidez } \\
\text { estomacal, flatulencias, } \\
\text { nervios }\end{array}$ & Infusión & $\begin{array}{l}\text { Órganos } \\
\text { externos }\end{array}$ & Bebida & Exótica \\
\hline Toronjil & Melissa officinalis L. & Laminaceae & $\begin{array}{l}\text { Nervios, Dolor de cabeza, } \\
\text { cólicos menstruales, } \\
\text { cansancio mental }\end{array}$ & Infusión & $\begin{array}{l}\text { Tallo- } \\
\text { Hoja }\end{array}$ & Bebida & Exótica \\
\hline Menta & Mentha piperita L. & Laminaceae & $\begin{array}{l}\text { Desordenes estomacales, } \\
\text { afecciones respiratorias, } \\
\text { anti espasmos }\end{array}$ & Infusión & $\begin{array}{l}\text { Órganos } \\
\text { externos }\end{array}$ & Bebida & Exótica \\
\hline Escancel & Aerva sanguinolenta $\mathrm{L}$. & Amaranthaceae & $\begin{array}{l}\text { Inflamación urinaria, } \\
\text { cáncer, mejora las defensas, } \\
\text { contra erupciones, anemia, } \\
\text { inflamación estomacal }\end{array}$ & $\begin{array}{l}\text { Infusión- } \\
\text { Zumo- } \\
\text { Macerado- } \\
\text { Triturado- } \\
\text { Emplasto }\end{array}$ & $\begin{array}{l}\text { Hoja y } \\
\text { Tallo }\end{array}$ & $\begin{array}{l}\text { Bebida-Uso } \\
\text { externo }\end{array}$ & Nativa \\
\hline Violeta & Viola arguta $\mathrm{K}$. & Violaceae & $\begin{array}{l}\text { Asma, pulmonía, gripes, } \\
\text { dolor de cabeza, fiebre }\end{array}$ & Infusión & $\begin{array}{l}\text { Flor y } \\
\text { Hoja }\end{array}$ & Bebida & Exótica \\
\hline Ruda & Ruta graveolens L. & Rutaceae & $\begin{array}{l}\text { Susto, dolor de cabeza, } \\
\text { fiebre, resfriado, nervios }\end{array}$ & $\begin{array}{l}\text { Infusión- } \\
\text { Triturado }\end{array}$ & $\begin{array}{l}\text { Hoja y } \\
\text { Flor }\end{array}$ & $\begin{array}{l}\text { Bebida-Uso } \\
\text { externo }\end{array}$ & Exótica \\
\hline Ortiga & Urtica urens L. & Urticaceae & $\begin{array}{l}\text { Circulación sangre, purifica } \\
\text { la sangre, ácido úrico, } \\
\text { reumatismo, dolor huesos, } \\
\text { estomacal y muscular, } \\
\text { cicatrizar ligaduras }\end{array}$ & $\begin{array}{l}\text { Infusión- } \\
\text { Zumo- } \\
\text { Macerado- } \\
\text { Triturado }\end{array}$ & Tópico & $\begin{array}{l}\text { Bebida-Uso } \\
\text { externo }\end{array}$ & Nativa \\
\hline Borraja & Borago officinalis L & Boraginaceae & $\begin{array}{l}\text { Problemas respiratorio, } \\
\text { gripe, tos seca, dolor de } \\
\text { estómago y vientre }\end{array}$ & Infusión & $\begin{array}{l}\text { Flor y } \\
\text { Hoja }\end{array}$ & Bebida & Exótica \\
\hline Malva & Malva sylvestris L. & Malvaceae & $\begin{array}{l}\text { Tos, gripe, infección } \\
\text { respiratoria y estomacal, } \\
\text { dolor muscular, renal, } \\
\text { infección estomacal, } \\
\text { desinflamante, estomacal, } \\
\text { antinflamatorio, } \\
\text { estreñimiento }\end{array}$ & Infusión & $\begin{array}{l}\text { Órganos } \\
\text { externos }\end{array}$ & Bebida & Exótica \\
\hline Hierbaluisa & $\begin{array}{l}\text { Cymbopogon citratus } \\
\text { S. }\end{array}$ & Poaeceae & $\begin{array}{l}\text { Tos, mejora defensa, } \\
\text { calmante, presión baja, } \\
\text { estimulante digestivo, } \\
\text { nervios, resfriado, dolor } \\
\text { estomacal, inflamación }\end{array}$ & Infusión & Tópico & Bebida & Exótica \\
\hline Sábila & Aloe vera $\mathrm{L}$ & Asphodelaceae & $\begin{array}{l}\text { Desinflamante, erupciones, } \\
\text { náuseas, analgésico } \\
\text { estomacal, cálculos hígado, } \\
\text { dolor renal }\end{array}$ & $\begin{array}{l}\text { Infusión-Jugo- } \\
\text { Macerado }\end{array}$ & Cristal & $\begin{array}{l}\text { Bebida-Uso } \\
\text { externo }\end{array}$ & Exótica \\
\hline Valeriana & Valeriana officinalis $\mathrm{L}$ & Valerianaceae & $\begin{array}{l}\text { Calmante nervioso, } \\
\text { insomnio }\end{array}$ & Infusión & Tópico & Bebida & Exótica \\
\hline Llantén & Plantago mayor $\mathrm{L}$ & Plantaginaceae & $\begin{array}{l}\text { Diuretico, dolor renal, } \\
\text { infección renal, problemas } \\
\text { respiratorios, gripe, } \\
\text { inflamación, parar } \\
\text { hemorragias }\end{array}$ & $\begin{array}{l}\text { Infusión- } \\
\text { Triturado }\end{array}$ & $\begin{array}{l}\text { Órganos } \\
\text { externos }\end{array}$ & $\begin{array}{l}\text { Bebida-Uso } \\
\text { externo }\end{array}$ & Exótica \\
\hline Eucalipto & Eucalyptus globulos L & Myrtaceae & $\begin{array}{l}\text { Gripe, asma, tos con flema, } \\
\text { problemas pulmonares, } \\
\text { afectaciones respiratorias, } \\
\text { resfriado, neumonía, } \\
\text { bronquitis }\end{array}$ & Infusión & Hoja & Inhalar-Bebida & Exótica \\
\hline Geranio & $\begin{array}{l}\text { Genranius } \\
\text { pelargonium } \mathrm{sp}\end{array}$ & Geraniaceae & $\begin{array}{l}\text { Cicatrizar heridas, } \\
\text { antiséptico en heridas, } \\
\text { cólicos menstruales }\end{array}$ & $\begin{array}{l}\text { Triturado- } \\
\text { Infusión }\end{array}$ & $\begin{array}{l}\text { Órganos } \\
\text { externos }\end{array}$ & $\begin{array}{l}\text { Uso externo- } \\
\text { Bebida-Baño }\end{array}$ & Exótica \\
\hline Fresno & Tecoma stans $\mathrm{L}$ & Bignoniacea & $\begin{array}{l}\text { Gripe, tos, bronquitis, } \\
\text { pulmonía }\end{array}$ & Infusión & Flor & Bebida & Nativa \\
\hline Ataco & Amaranthus muricatus & Amaranthaceae & Colicos menstruales, & Infusión & Órganos & Bebida-Baños & Nativa \\
\hline
\end{tabular}




\begin{tabular}{|c|c|c|c|c|c|c|c|}
\hline $\begin{array}{l}\text { Nombre } \\
\text { común }\end{array}$ & $\begin{array}{l}\text { Nombre } \\
\text { científico }\end{array}$ & Familia & Uso medicinal & $\begin{array}{l}\text { Método } \\
\text { preparación }\end{array}$ & $\begin{array}{c}\text { Parte } \\
\text { utilizada }\end{array}$ & $\begin{array}{c}\text { Vía } \\
\text { administración } \\
\end{array}$ & Origen \\
\hline & & & $\begin{array}{l}\text { desinflamante, vías } \\
\text { urinarias, cólicos } \\
\text { menstrúales, gripe, limpiar } \\
\text { organismo }\end{array}$ & & externos & & \\
\hline $\begin{array}{l}\text { Cola de } \\
\text { caballo }\end{array}$ & Equisetum arvense $\mathrm{L}$ & Equisetaeceae & $\begin{array}{l}\text { Problemas renales, gripe, } \\
\text { desinflamación vías } \\
\text { urinarias y renal }\end{array}$ & Infusión & $\begin{array}{l}\text { Tallo- } \\
\text { Hoja }\end{array}$ & Bebida & Exótica \\
\hline Cascarilla & $\begin{array}{l}\text { Cinchona } \\
\text { pubescens Vahl }\end{array}$ & Rubiaceae & $\begin{array}{l}\text { Pulmonia, vías } \\
\text { respiratorias, gripe, } \\
\text { neumonía, tos }\end{array}$ & Infusión & Tallo & Bebida & Nativa \\
\hline $\begin{array}{l}\text { Diente de } \\
\text { León }\end{array}$ & Taraxacum officinale $\mathrm{L}$ & Asteraceae & $\begin{array}{l}\text { Desinflamante de hígado, } \\
\text { circulación de la sangre, } \\
\text { dolor garganta y renal }\end{array}$ & Infusión & $\begin{array}{l}\text { Órganos } \\
\text { externos }\end{array}$ & Bebida & Exótica \\
\hline Buscapina & Parietaria officinalis $\mathrm{L}$ & Urticaceae & $\begin{array}{l}\text { Colicos menstruales, dolor } \\
\text { estomacal y garganta }\end{array}$ & Infusión & Hoja & Bebida & Exótica \\
\hline Romero & Rosmarinus Officinalis & Laminaceae & $\begin{array}{l}\text { Dolor de cabeza, hidratante } \\
\text { en fiebre, alergias }\end{array}$ & $\begin{array}{l}\text { Infusión- } \\
\text { Triturado- } \\
\text { Macerado }\end{array}$ & Hoja & $\begin{array}{l}\text { Bebida-Baño- } \\
\text { Uso externo }\end{array}$ & Exótica \\
\hline Matico & Piper aduncum $\mathrm{L}$ & Piperacea & $\begin{array}{l}\text { Dolor de hueso, hígado } \\
\text { graso, des inflamatorio } \\
\text { heridas, picaduras }\end{array}$ & $\begin{array}{l}\text { Infusión- } \\
\text { Cocción }\end{array}$ & Hoja & $\begin{array}{l}\text { Uso externo- } \\
\text { Bebida }\end{array}$ & Nativa \\
\hline Verbena & Verbena officinalis L & Verbenaceae & $\begin{array}{l}\text { Dolor garganta, gripe, } \\
\text { diabetes }\end{array}$ & Infusión & Hoja & Bebida & Exótica \\
\hline Pena-Pena & Fuchsia vulcanica $\mathrm{L}$ & Onagraceae & $\begin{array}{l}\text { Nervios, presión alta, } \\
\text { desinflamación de vías } \\
\text { urinarias }\end{array}$ & Infusión & Flor-Hoja & Bebida & Nativa \\
\hline Clavel & Dianthus caryphyllus L & Caryophyllaceae & $\begin{array}{l}\text { Trastornos mentales, } \\
\text { nervios, desinflamante } \\
\text { urinario }\end{array}$ & Infusión & Flor-Hoja & Bebida & Nativa \\
\hline Mortiño & Solanum nigrescens $\mathrm{M}$ & Solanaceae & $\begin{array}{l}\text { Infección estomacal-gripe, } \\
\text { dolor de cabeza y } \\
\text { estomacal }\end{array}$ & $\begin{array}{l}\text { Infusión- } \\
\text { Zumo }\end{array}$ & $\begin{array}{l}\text { Órganos } \\
\text { externos }\end{array}$ & Bebida & Nativa \\
\hline Orégano chino & Origanum vulgare $\mathrm{L}$ & Laminaceae & $\begin{array}{l}\text { Dolor estomacal- } \\
\text { antinflamatorio, cólicos, } \\
\text { menstrúales }\end{array}$ & Infusión & Hoja & Bebida & Exótica \\
\hline Malva blanca & $\begin{array}{l}\text { Sphaeralcea } \\
\text { bonariensis C }\end{array}$ & Malvaceae & $\begin{array}{l}\text { Infección bucal, fiebre, } \\
\text { inflamación }\end{array}$ & Infusión & Flor-Hoja & Bebida & Exótica \\
\hline Cedrón & Lippia citriodora $\mathrm{K}$ & Verbenaceae & Nervios, tos, insomnio & Infusión & Hoja & Bebida & Nativa \\
\hline Gañil & $\begin{array}{l}\text { Oreocallis grandiflora } \\
\mathrm{L}\end{array}$ & Protaceae & Dolor renal & Infusión & Flor & Bebida & Nativa \\
\hline Tipo & $\begin{array}{l}\text { Minthostachys mollis } \\
\mathrm{G}\end{array}$ & Laminaceae & Gripe, dolor de estomago & Infusión & Hoja & Bebida & Nativa \\
\hline Mentol & $\begin{array}{l}\text { Cornus peruviana J.F. } \\
\text { Macbr }\end{array}$ & Cornaceae & Tos & Infusión & $\begin{array}{l}\text { Hoja- } \\
\text { Tallo }\end{array}$ & Bebida & Nativa \\
\hline Aliso & Alnus acuminata $\mathrm{K}$ & Betulaceae & $\begin{array}{l}\text { Tos, fiebre, circulación de } \\
\text { la sangre, desinflamante }\end{array}$ & Infusión & $\begin{array}{l}\text { Tallo- } \\
\text { Hoja }\end{array}$ & Bebida & Nativa \\
\hline Rosa Blanca & Rosa centifolia $\mathrm{L}$ & Rosaceae & Inflamación urinaria & Infusión & Hoja & Bebida & Exótica \\
\hline Nogal & Uglans neotropica D & Juglandaceae & $\begin{array}{l}\text { Resfriado, calcio para } \\
\text { huesos }\end{array}$ & Infusión & Hoja & Bebida & Nativa \\
\hline Guaviduca & $\begin{array}{l}\text { Piper carpunya Ruiz y } \\
\text { Pav }\end{array}$ & Piperacea & Asma, gastritis, cáncer & Infusión & Hoja & Bebida & Nativa \\
\hline Ginseng & Panax ginseng & Araliaceae & Mejora defensa, tos & $\begin{array}{l}\text { Macerado- } \\
\text { Infusión }\end{array}$ & $\begin{array}{l}\text { Tallo- } \\
\text { Hoja }\end{array}$ & Bebida & Exótica \\
\hline Naranja & Citrus aurantium $\mathrm{L}$ & Rutaceae & Mejora defensa, gripe & Infusión & Hoja & Bebida & Exótica \\
\hline Ciprés & $\begin{array}{l}\text { Cupressus } \\
\text { Sempervirens L. }\end{array}$ & Cupresaceae & Bronquitis & Cocción & Hoja & Inhalación & Exótica \\
\hline Chilca & $\begin{array}{l}\text { Aristeguietia } \\
\text { cacalioides } \mathrm{K}\end{array}$ & Asteraceae & Presión baja & Cocción & Hoja & Uso externo & Nativa \\
\hline Altamisa & $\begin{array}{l}\text { Ambrosia arborescens } \\
\text { Mill }\end{array}$ & Asteraceae & $\begin{array}{l}\text { Dolor estomacal, cólicos } \\
\text { menstrúales }\end{array}$ & Infusión & Hoja & Bebida & Nativa \\
\hline Pensamiento & Viola odorata $\mathrm{L}$ & Violaceae & Nervios & Infusión & Flor & Bebida & Exótica \\
\hline Malva Olorosa & $\begin{array}{l}\text { Pelargonium } \\
\text { odoratissimun } \mathrm{L}\end{array}$ & Geraniaceae & $\begin{array}{l}\text { Frio, desinflama vías } \\
\text { urinarias }\end{array}$ & Infusión & Flor & Bebida & Exótica \\
\hline Ajo & Allium sativum $\mathrm{L}$ & Amaryllidaceae & Secar fuegos, gripe & $\begin{array}{l}\text { Triturado- } \\
\text { Infusión }\end{array}$ & Bulbo & Bd-Uxt & Exótica \\
\hline Jengibre & Zingiber officinale & Zingiberaceae & Dolor de garganta, & Macerado & Raíz & Bebida & Exótica \\
\hline
\end{tabular}




\begin{tabular}{|c|c|c|c|c|c|c|c|}
\hline $\begin{array}{l}\text { Nombre } \\
\text { común }\end{array}$ & $\begin{array}{l}\text { Nombre } \\
\text { científico }\end{array}$ & Familia & Uso medicinal & $\begin{array}{l}\text { Método } \\
\text { preparación }\end{array}$ & $\begin{array}{c}\text { Parte } \\
\text { utilizada }\end{array}$ & $\begin{array}{c}\text { Vía } \\
\text { administración } \\
\end{array}$ & Origen \\
\hline Tomillo & Thymus vulgarius $\mathrm{L}$ & Laminaceae & $\begin{array}{l}\text { afectación respiratoria } \\
\text { Antiseptico, dolor } \\
\text { estomacal }\end{array}$ & Infusión & Hoja & Bebida & Exótica \\
\hline Pulmonaria & $\begin{array}{l}\text { Pulmonaria officinalis } \\
\mathrm{L}\end{array}$ & Boraginaceae & Pulmonia & Infusión & $\begin{array}{l}\text { Órganos } \\
\text { externos }\end{array}$ & Bebida & Exótica \\
\hline Chuquiragua & Chuquiraga jussieui & Asteraceae & Gripe & Infusión & Flor & Bebida & Nativa \\
\hline Perlilla & Perilla frutescens $\mathrm{L}$ & Laminaceae & Gripe & Infusión & Hoja-Raíz & Bebida & Exótica \\
\hline Colín Culén & Psoralea glandulosa & Fabacea & Dolor estomacal & Infusión & Hoja & Bebida & Nativa \\
\hline Paico & $\begin{array}{l}\text { Dysphania } \\
\text { ambrosioides } \mathrm{L}\end{array}$ & Amaranthaceae & Parasitos & In & Raíz & Bebida & Nativa \\
\hline Sauco & Cestrum peruvianum & Solanaceae & Dolor estomacal y cabeza & $\begin{array}{l}\text { Infusión- } \\
\text { Zumo }\end{array}$ & Flor-Hoja & Bebida & Nativa \\
\hline Boldo & $\begin{array}{l}\text { Oreocallis grandiflora } \\
\mathrm{L}\end{array}$ & Protaceae & Dolor estomacal & Infusión & $\mathrm{Hj}$ & Bebida & Nativa \\
\hline Congona & $\begin{array}{l}\text { Peperomia rotundata } \\
\mathrm{K}\end{array}$ & Piperacea & Colicos menstrúales & Infusión & $\mathrm{Hj}$ & $\mathrm{Bd}$ & Nativa \\
\hline Berro & Nasturtium officinale & Brassicaceae & Vitaminas, desparasitaste & Jugo & $\mathrm{Tll}-\mathrm{Hj}$ & $\mathrm{Bd}$ & Exótica \\
\hline Marco & $\begin{array}{l}\text { Ambrosia arborescens } \\
\text { Mill }\end{array}$ & Asteraceae & Artritis, resfriado & Infusión & Flor & $\mathrm{Bd}$ & Exótica \\
\hline Papa silvestre & $\begin{array}{l}\text { Solanum tuberosum } \\
\text { silvestre } \mathrm{L}\end{array}$ & Solanaceae & Prostata, gastritis & $\begin{array}{l}\text { Cocción- } \\
\text { Infusión }\end{array}$ & $\begin{array}{l}\text { Hoja- } \\
\text { Tubérculo }\end{array}$ & $\begin{array}{l}\text { Comida- } \\
\text { Bebida }\end{array}$ & Nativa \\
\hline Zarzaparrilla & Smilax aspera $\mathrm{L}$ & Smilacaceae & Riñones, próstata & Infusión & Raíz & Bebida & Exótica \\
\hline Limón & Citrus limonum L & Rutaceae & Gripe, fiebre & Infusión & Fruto & Bebida & Exótica \\
\hline Cebolla & Allium cepa $\mathrm{L}$ & Amaryllidaceae & Dolor estómago, gastritis & Infusión & Raíz & Bebida & Exótica \\
\hline Oreja de burro & Stachys lanta $\mathrm{K}$ & Laminaceae & Tos, fiebre & Infusión & Hoja & Bebida & Exótica \\
\hline Laurel & Laurus nobilis L & Lauraceae & Resfriado & Infusión & Hoja & Bebida & Exótica \\
\hline Sauco negro & Sumbucus nigra $\mathrm{L}$ & Adoxaceae & Fiebre, dolor de cabeza & Zumo & Hoja & Uso externo & Exótica \\
\hline Poleo & Clinopodium brownei & Laminaceae & Dolor de cabeza & Emplasto & Hoja & Uso externo & Nativa \\
\hline Pedorrera & Galinsoga sp & Asteraceae & Dolor estomacal & Infusión & Hoja & Bebida & Nativa \\
\hline Aguacate & Persea americana Mill & Lauraceae & Dolor de muela & Triturado & Hoja & Comida & Exótica \\
\hline Ibuprofeno & Plectranthus ornatus & Laminaceae & Infección estomacal & Infusión & Hoja & Bebida & Exótica \\
\hline Dulcamara & Kalanchoe pinnata $\mathrm{L}$ & Crassulaceae & Antinflamatorio & Macerado & $\begin{array}{l}\text { Órganos } \\
\text { externos }\end{array}$ & Bebida & Exótica \\
\hline Noni & Morínda citrifolia $\mathrm{L}$ & Rubiaceae & Antioxidante & Jugo & Fruto & Bebida & Exótica \\
\hline Stevia & Stevia rebaudiana & Asteraceae & Diabetes & Infusión & Hoja & Bebida & Exótica \\
\hline Linaza & Linum usitatissimum $\mathrm{L}$ & Linaceae & Diuretico & Infusión & Hoja & Bebida & Exótica \\
\hline Muerdago & Viscum album $\mathrm{L}$ & Santalaceae & Presión & Infusión & Hoja & Bebida & Exótica \\
\hline Apio & Apium graveolens $\mathrm{L}$ & Apiaceae & Dolor estomacal & Jugo & Tallo & Bebida & Exótica \\
\hline Achojcha & Cyclanthera pedata $\mathrm{L}$ & Cucurbitaceae & Dolor de oido & Cocción & Fruto & Uso externo & Nativa \\
\hline Chanca Piedra & Phyllanthus niruri L & Phyllanthaceae & Dolor renal & Infusión & $\begin{array}{l}\text { Órganos } \\
\text { externos }\end{array}$ & Bebida & Nativa \\
\hline Amapola & Papaver rhoeas $\mathrm{L}$ & Papaveraceae & Inflamación del vientre & Infusión & Flor & Bebida & Exótica \\
\hline Mejorona & Origanum majorana $\mathrm{L}$ & Laminaceae & Gripe & Infusión & Hoja & Bebida & Exótica \\
\hline Insulina & Cissus verticillata $\mathrm{L}$ & Vitaceae & Calmante & Infusión & Hoja & Bebida & Nativa \\
\hline Congona & Brosimum alicastrum $\mathrm{S}$ & Moraceae & Nervioso & Zumo & $\begin{array}{l}\text { Hoja- } \\
\text { Tallo }\end{array}$ & Bebida & Nativa \\
\hline Inojo & Foeniculum vulgare $\mathrm{M}$ & Apiaceae & Leche materna & Infusión & Hoja & Bebida & Exótica \\
\hline Albahaca & Ocimum basilicum $\mathrm{L}$ & Laminaceae & Leche materna & Infusión & Hoja & Bebida & Exótica \\
\hline Consuelda & $\begin{array}{l}\text { Symphytum officinale } \\
\mathrm{L}\end{array}$ & Boraginaceae & Cicatrizar heridas & Triturado & $\begin{array}{l}\text { Órganos } \\
\text { externos }\end{array}$ & Uso externo & Exótica \\
\hline Té silvestre & Bidens aurea $\mathrm{S}$ & Asteraceae & Diarrea & Infusión & $\begin{array}{l}\text { Órganos } \\
\text { externos }\end{array}$ & Bebida & Nativa \\
\hline Chichiru & Bidens pilosa $\mathrm{L}$ & Asteraceae & Resfriado & Infusión & Hoja & Bebida & Nativa \\
\hline Cardiaca & Leonurus cardiaca $\mathrm{L}$ & Laminaceae & Nervioso & Infusión & Hoja & Bebida & Exótica \\
\hline Hierbabuena & Mentha spicata $\mathrm{L}$ & Laminaceae & Resfrio & Triturado & Hoja & Comida & Exótica \\
\hline Anís & $\begin{array}{l}\text { Pimpinella anisum } \\
\mathrm{L}\end{array}$ & Apiaceae & Dolor estomacal & Infusión & $\begin{array}{l}\text { Órganos } \\
\text { externos }\end{array}$ & Bebida & Exótica \\
\hline Maíz & Zea mays $\mathrm{L}$ & Poaeceae & Dolor estomacal & Infusión & $\begin{array}{l}\text { Órganos } \\
\text { externos }\end{array}$ & Bebida & Exótica \\
\hline Col & Brassica oleracea $\mathrm{L}$ & Brassicaceae & Inflamación estomacal & Infusión & Estigmas & Bebida & Exótica \\
\hline Lechuga & Lactuca sativa $\mathrm{L}$ & Asteraceae & Gastritis & Infusión & Hoja & Bebida & Exótica \\
\hline Santa María & $\begin{array}{l}\text { Tanacetum balsamita } \\
\mathrm{L}\end{array}$ & Asteraceae & Inflamación de hígado & Infusión & Hoja & Bebida & Exótica \\
\hline
\end{tabular}

